\title{
Predicting the Analog Integrated Circuit Performance Using Indirect Measurements Based on Simulations
}

\author{
Senan Alraho', Qummar Zaman, Andreas König ${ }^{1}$ \\ ${ }^{1}$ Lehrstuhl Integrierte Sensorsysteme (ISE), TU Kaiserslautern, Deutschland, \\ aube@eit.uni-kl.de
}

\begin{abstract}
Summary:
This paper presents a low-cost indirect measurement method to predict the performance of analog integrated circuits (ICs). The method is based on integrating simple sensors near to the main circuit to capture circuit properties under different conditions expected by real fabrication. Monte Carlo with corner simulation results is used to train neural network for modeling and mapping the sensor outputs to the design output performance. The achieved correlation performance metrics is $91.68 \%$. The circuit is designed using AMS $0.35 \mu \mathrm{m}$ technology and Cadence tools.
\end{abstract}

Keywords: Neural Network, Artificial Intelligence, Indirect Measurements, Non-intrusive sensors.

\section{Background, Motivation an Objective}

The merging of $\mathrm{Al}$ and $\mathrm{ML}$ with other developing technologies, such as industrial internet of things I(i)OT and cyber-physical production systems (CPPS) is empowering the significant revolution in the industrial domain [1] known as industrial 4.0 [2]. In industry 4.0 and loTs devices, smart integrated sensors perform a central role in data generation [1]. Normally the performance of smart sensory electronics (SSE) is decayed with the passage of time [3]. To address the aging and process variations effects, commonly, analog ICs are overdesigned, that leads to consume more power and or larger chip area. Nevertheless, ML in SSE will enable the self-x (self-optimize, self-calibration, selfhealing, etc.) properties [4]. One of the primary calibration methods is designing the analog ICs with controllable tuning knobs and performance measurement set-up [5] for chip performance monitoring [6]. So, the main objective in this research work is to find out a cost-effective indirect performance evaluation method (IMs) for SSE toward reducing the number of real expensive chip measurements.

\section{Proposed Methodology}

The block diagram of the proposed IMs is presented in Fig. 1, the non-intrusive sensors (NS) are integrated on chip in close proximity to the main design under test (DUT) to face the same conditions imposed on the DUT, that is PVT variations (process, voltage, temperature). An artificial neural network (ANN) is used to map NS and DUT performance and creates accurate regression model to indirectly and more easily predict DUT performance based on the sensors data. The flow diagram of the proposed measurement approach is depicted in Fig. 2.

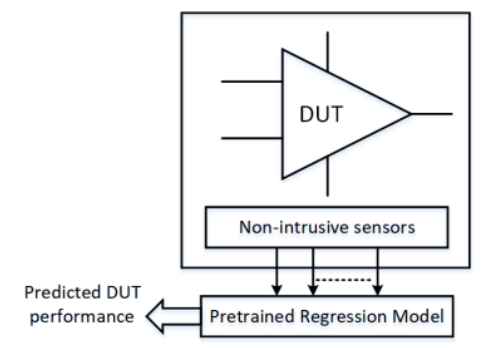

Fig. 1: Block diagram of the proposed IMs method

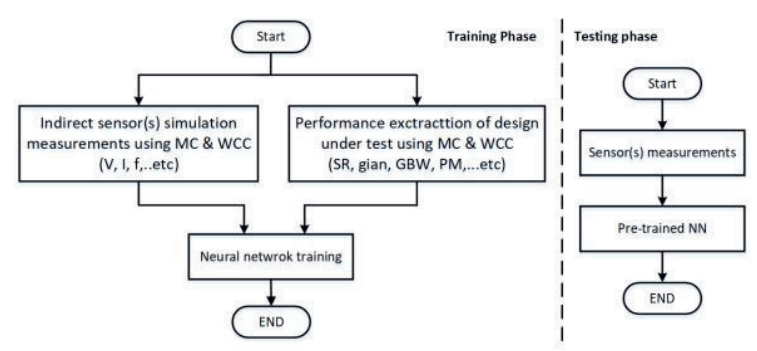

Fig. 2: Flow chart of the proposed IMs methodology

During training phase, both DUT and NS are simulated and subjected to the same PVT condition using combination of worse case corners (WCC) and Monte Carlo (MC) with enough samples (200 samples) to accurately capture real fabrication process profile. $80 \%$ of data samples are randomly selected to train the ANN while the remaining $20 \%$ are used to evaluate its performance. In the testing phase, the data provided by NS to the pre-trained ANN is used to indirectly predict the DUT performance

A single-ended operational amplifier is used as DUT in this experiment to verify the proposed 
concept. The measurements quantities of the DUT are slew rate, DC gain, gain-bandwidth product, and phase margin. The remaining simple characteristics are left for the real measurement because it will consume considerable time during simulation. A feasible solution is to increase computational resources to cover more tests by simulation. The clock generator based on ring oscillator (RO) circuit shown in Fig. 3 is designed as PVT monitoring circuit.

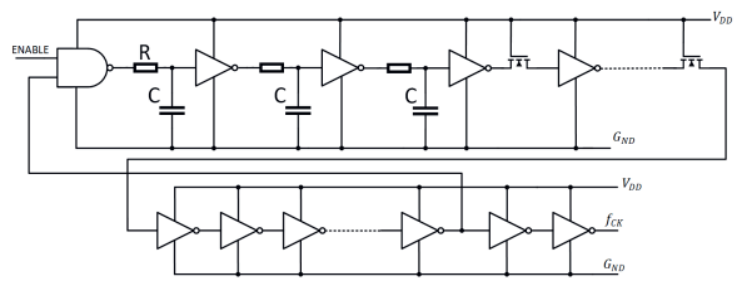

Figure 3: Ring oscillator for process monitoring.

RO shares almost the same circuit devices of the DUT to increase data correlation between both and thus improving the ANN modelling and prediction accuracy. The first test result shows weak prediction level of the ANN with adjusted $R$ squared value (ARS) of $30.48 \%$. It concludes that although $\mathrm{RO}$ alone is showing good sensitivity to the PVT variations, however, its performance is mostly dominated by $\mathrm{RC}$ components, thus not providing enough correlation information with the DUT to the ANN. Hence, additional NS with different sensitivities to PVT is required to further enhance ANN modelling. Authors in [7] presented a circuit to monitor the transistors threshold voltage (Vth) variation with the process by reading the voltage drop on PMOS diode connected transistor working in the weak inversion. Though, we count that is not enough to monitor Vth of PMOS only while the NMOS can go in opposite process corner. Hence, we proposed a modified version of this circuit as shown in Fig. 4 to monitor both of NMOS and PMOS Vth variations.

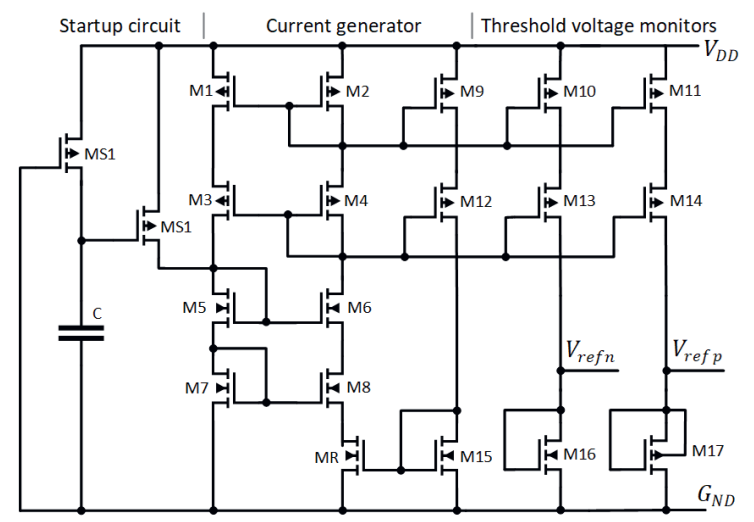

Figure 4: Threshold voltage monitor circuit.

The resistor-less current biasing circuit [8] is used to generate reference current insensitive to supply voltage and has small positive tem- perature coefficient. All transistors in the circuit work in the subthreshold region, except for MR that is in the triode region functioning as MOS resistor. By including PMOS threshold voltage readings from Vrefp together with the $\mathrm{RO}$ frequency data, the measurement prediction is improved with ARS of $70.5 \%$. In the last experiment the Vrefn data is added and the prediction accuracy improved significantly with ARS equal to $91.68 \%$. The performance of the proposed methodology is illustrated graphically in Fig. 5. In the future, we are working to have all types of NS outputs in digital form to have more immune outputs to the observer noise.
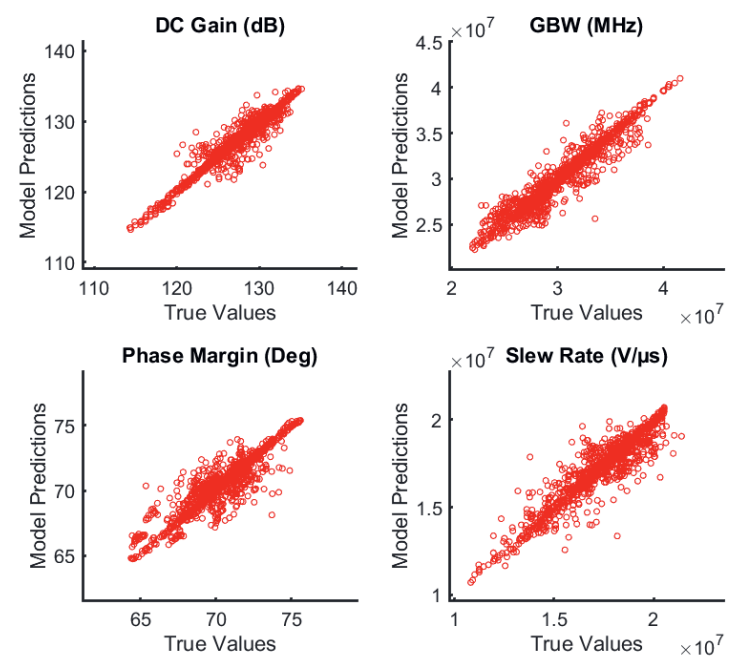

Figure 5: Scatter plot of the predicted and true values.

\section{References}

[1] Diez-Olivan, Alberto, et al. "Data fusion and machine learning for industrial prognosis: Trends and perspectives towards Industry 4.0." Information Fusion 50 (2019).

[2] Henning, Kagermann. "Recommendations for implementing the strategic initiative INDUSTRIE 4.0." (2013).

[3] Lee, Sanghoon, et al. "A built-in self-test and in situanalog circuit optimization platform." IEEE Transactions on Circuits and Systems (2018)

[4] Andraud, Martin, Haralampos-G. Stratigopoulos, and Emmanuel Simeu. "One-shot non-intrusive calibration against process variations for analog/RF circuits." IEEE TCS 2016.

[5] Andraud, Martin, Haralampos-G. Stratigopoulos, and Emmanuel Simeu. "One-shot non-intrusive calibration against process variations for analog/RF circuits." IEEE TCS 2016.

[6] In chip monitoring, telemetry and analytics", 2020 (accessed 2020). URL https://www.moortec.com.

[7] Osaki, Yuji, et al. "Delay-compensation techniques for ultra-low-power subthreshold CMOS digital LSIs." IEEE, 2009 52nd IEEE International Midwest Symposium on Circuits and Systems.

[8] Oguey, Henri J., and Daniel Aebischer. "CMOS current reference without resistance." IEEE Journal of Solid-State Circuits 32.7 (1997). 